Research Paper

\title{
Eifects of a Sliding Plate on Morphology of the Epiphyseal Plate in Goat Distal Femur
}

\author{
Da-sheng LIN, Ke-jian LIAN ${ }^{\bowtie}$, Jia-yuan HONG, Zhen-qi DING, Wen-liang ZHAI
}

Department of Orthopaedic Surgery, the Affiliated Southeast Hospital of Xiamen University, Orthopaedic Center of People's Liberation Army, Zhangzhou 363000, China.

$\triangle$ Corresponding author: Ke-jian LIAN Department of Orthopaedic Surgery, The Affiliated Southeast Hospital of Xiamen University, Orthopaedic Center of People's Liberation Army, Zhangzhou 363000, China. Tel: +86-596-2931538; Fax: +86-596-2931538; Email: 16621021@qq.com

(C) Ivyspring International Publisher. This is an open-access article distributed under the terms of the Creative Commons License (http://creativecommons.org/ licenses/by-nc-nd/3.0/). Reproduction is permitted for personal, noncommercial use, provided that the article is in whole, unmodified, and properly cited.

Received: 2011.10.08; Accepted: 2012.01.18; Published: 2012.02.05

\begin{abstract}
The aim of this study was to observe the effects of a sliding plate on the morphology of the epiphyseal plate in goat distal femur. Eighteen premature female goats were divided randomly into sliding plate, regular plate and control groups. Radiographic analysis and histological staining were performed to evaluate the development of epiphyseal plate at 4 and 8 weeks after surgery. In the sliding plate group, the plate extended accordingly as the epiphyseal plate grows, and the epiphyseal morphology was kept essential normal. However, the phenomenon of the epiphyseal growth retardation and premature closure were very common in the regular plate group. In addition, the sliding plate group exhibited more normal histologic features and Safranin $O$ staining compared to the regular plate group. Our results suggest that the sliding plate can provide reliable internal fixation of epiphyseal fracture without inhibiting epiphyseal growth.
\end{abstract}

Key words: epiphyseal plate, sliding, internal fixation, morphology

\section{Introduction}

The growth characteristics of epiphyseal plate determine that the treatment method of epiphyseal fracture is different from that of normal fracture. The ideal treatment method should provide adequate stability to permit early mobilization, preserve or optimize fracture biology without inhibiting epiphyseal growth, avoid serious complications, and achieve these goals in a cost-effective manner [1-3]. Fractures of the distal femoral growth plate are remarkable in that are third most common epiphyseal fracture in children (after wrist and ankle), yet they carry a risk of growth disturbance in up to $90 \%$ of cases $[4,5]$. Salter-Harris system is used to classify these injures [6]. Salter-Harris type-I and II fractures can be treated with closed reduction and percutaneous pin or screw fixation. Salter-Harris type-III and IV fractures are best treated with an open reduction and percutaneous or internal fixation, usually with Kirschner wires or screws that do not cross the physis $[7,8]$. However, we often encounter the complex fractures of the distal femoral growth plate in the clinic, and the Kirschner wires or screws fixation itself may not be reliable. Regular plate fixation can be relied on for fixation but may retard vertical growth of the bones and lead to the femoral valgus deformity and leg-length discrepancy [9]. In order to tackle these problems, we designed a sliding plate to observe the effects of it on the morphology of the epiphyseal plate, and develop its feasibility on providing reliable internal fixation of epiphyseal fracture without inhibiting epiphyseal 
growth.

\section{Methods}

\section{Designing of sliding plate}

In collaboration with Double Engine Medical Material Co Ltd (Xiamen, China), we have designed a sliding plate, for which we have obtained a patent (patent number: 200620009578.4). this internal device is made of titanium alloy and is a transformational approach based on regular anatomical plate. To realize the vertical slide function, the internal device is composed of two parts. The head, $68 \mathrm{~mm}$ in length, 13 $\mathrm{mm}$ in width and $4 \mathrm{~mm}$ in thickness, is used to fix the femoral condyle of goat, and the body, $63.5 \mathrm{~mm}$ in length, $9.3 \mathrm{~mm}$ in width and $2.5 \mathrm{~mm}$ in thickness, is used to fix the diaphysis. The head has a drawer-like slot along which the body portion can slide (Fig. 1).

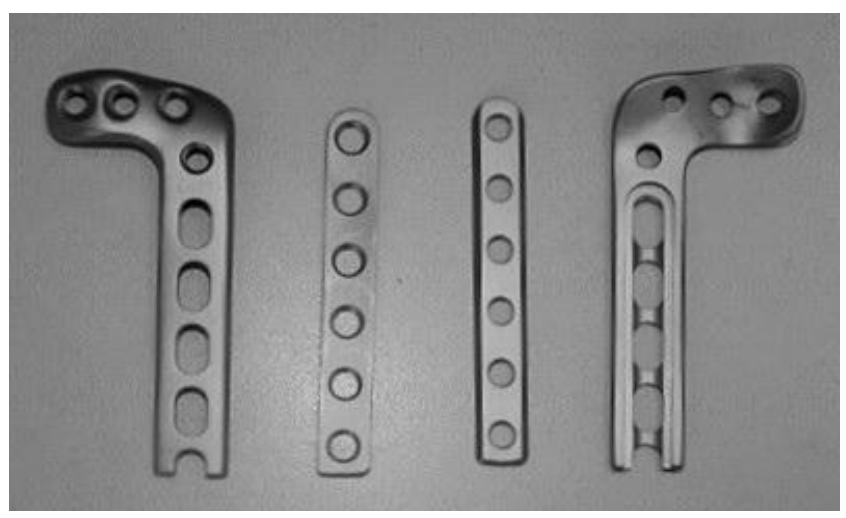

Fig. I Picture for the sliding plate.

\section{Experimental animals and grouping}

All animal experimental procedures were approved and in accordance with the Institutional Animal Care and Use Committee of the authors' institution. Eighteen female, 8-10 weeks old goats were maintained in the animal care facility for 10 days to become acclimated to diet, water, and housing under a 12 hour/12 hour light/dark cycle. All goats were divided randomly into sliding plate group $(n=6)$, regular plate group $(n=6)$ and control group $(n=6)$. In sliding plate group were fixed with sliding plate, regular plate group were fixed with regular plate and in control group were exposed without internal fixation.

\section{Surgical procedure}

All surgeries were performed by the first author under endotracheal intubation and general anesthe- sia. The femoral condyle was exposed and the cartilage membrane surrounding the physis was protected. A Kirschner wire with a diameter of $1.5 \mathrm{~mm}$ was introduced at the lateral condyle, which was located using intra-operative $\mathrm{X}$-ray radiography in order to ascertain the relative location of the Kirschner wire and physis and to avoid damaging the physis itself. Sliding plate was introduced at the lateral condyle. Using the same method, regular plates were placed respectively. In control group were exposed without internal fixation. At two time points (postoperative 4 and 8 weeks), three animals were obtained for positive and lateral femur X-ray film on operational femur in each group respectively, and the histological staining was performed to evaluate the development of epiphyseal plate.

\section{Light microscope examination}

The fresh bone specimens were fixed with formaldehyde, decalcified, and embedded in paraffin. Each slide was cut to $5 \mu \mathrm{m}$ thickness, and routine hematoxylin and eosin (HE) staining was performed. Under light microscopy, all the tissue slides were put into a VIDAS automatic image analysis machine. The thickness of the epiphyseal was measured under $40 x$ magnification, and proliferation cells and hypertrophic cells were counted.

\section{Histochemical analysis with Safranin 0}

Specimens were isolated from the distal femur, fixed with $20 \%$ neutral formaldehyde, and decalcified with decalcification buffer. The femoral condyle was cut down from the center. After a series of dehydration procedures, routine slides were cut from paraffin-embedded tissues at $5 \mu \mathrm{m}$ thicknesses individually. All slides from the total samples (including those taken at different times) were stained under the same conditions and with Safranin O. Safranin O binds to proteoglycan, so a stronger red staining with Safranin $\mathrm{O}$ indicates a higher amount of proteoglycan, from which we can find the concentration of cytosolic proteoglycan and study the growth of cartilage cells.

\section{Statistical analysis}

The data was analyzed using SPSS13.0 statistics software. $P<0.05$ was considered to be statistically significant, $P<0.01$ was considered to be extremely statistically significant.

\section{Results}

\section{Radiographic analysis}

Radiographic analysis of the femurs in the goats revealed that the sliding plate group could be ex- 
tended as the femur grew. The epiphyseal morphology in the sliding plate group was kept essential normal. However, the phenomenon of the epiphyseal growth retardation and premature closure were very common in the regular plate group (Fig. 2 and Fig. 3).
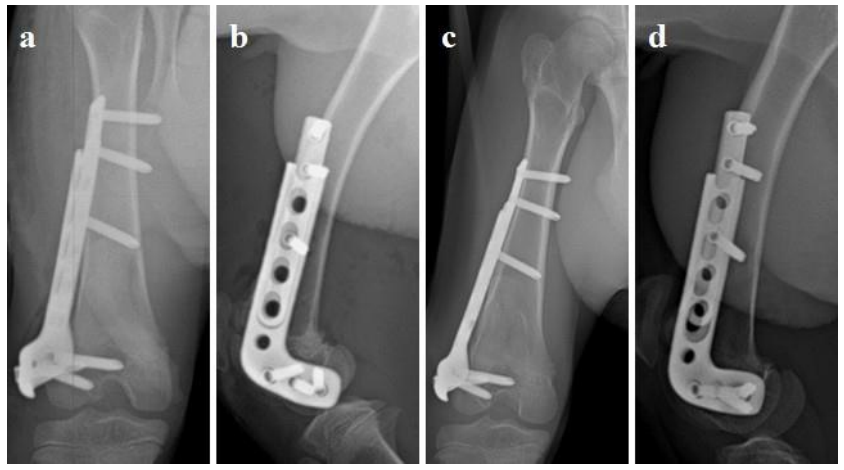

Fig. 2 Experimental goat distal femur was fixed with sliding plate. The sliding plate extended accordingly as the epiphyseal plate grows. a: Posterior-anterior radiograph of goat femur (postoperative 0 day). b: Lateral radiograph of goat femur (postoperative 0 day). c: Posterior-anterior radiograph of goat femur (postoperative 4 weeks). d: Lateral radiograph of goat femur (postoperative 4 weeks).
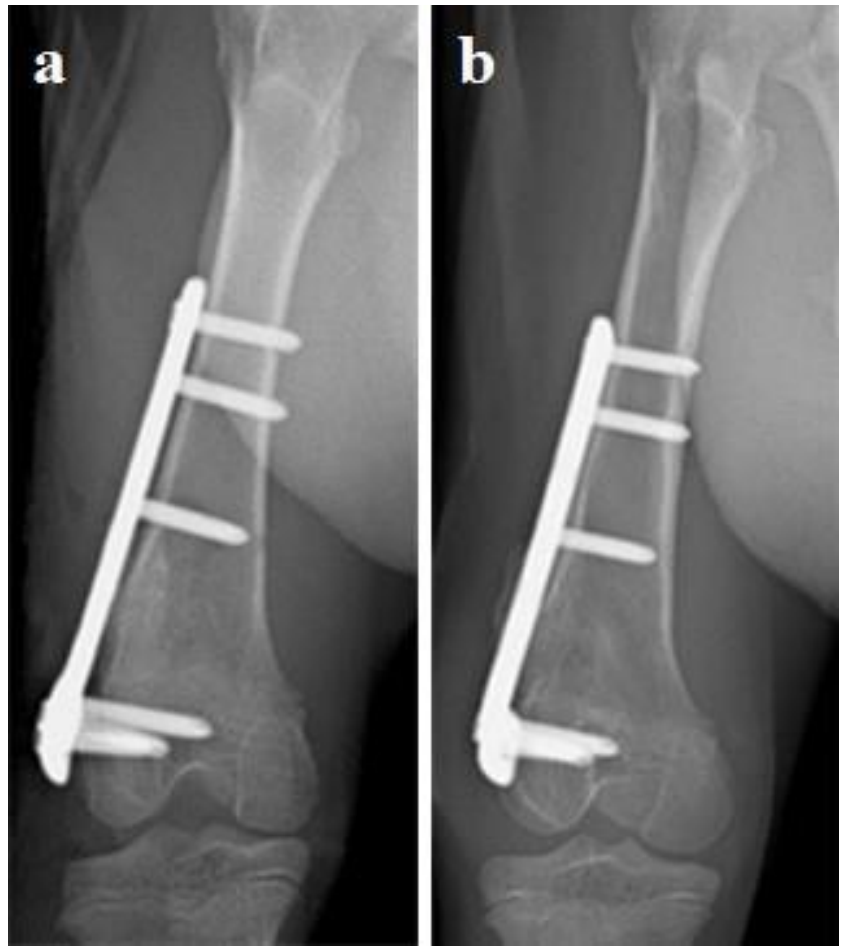

Fig. 3 Experimental goat distal femur was fixed with regular plate. The plate and screws restricted the epiphyseal unequal growth. a: Posterior-anterior radiograph of goat femur (postoperative 0 day). b: Posterior-anterior radiograph of goat femur (postoperative 4 weeks).

\section{Light microscope examination}

The HE staining examination at 4 weeks and 8 weeks after surgery indicated that cartilage cells in both the sliding plate group and the control group were longitudinally oriented and extended upwards in an orderly fashion. Vigorous mitotic processes were observed in the upper cell column, with abundant hypertrophic cells. In the regular plate group, the cells were narrower and disordered, and cartilage cells were less ordered in comparison to both the sliding plate group and the control group. At 4 weeks and 8 weeks after surgery, There were significant differences in the epiphyseal plate thicknesses, proliferation cell counts and hypertrophic cell counts in the sliding plate group vs. the regular plate group $(P<0.05)$, and there were no significant difference between the sliding plate group and the control group $(P>0.05)$. Results are shown in Table 1 and Fig. 4.

\section{Safranin $\mathbf{O}$ histochemistry staining}

Cartilage cells and cytosol from all the groups were stained with Safranin O, which exhibited a red color. Compared with tissues taken at 4 weeks or 8 weeks after surgery, Safranin O staining was significantly stronger in the sliding plate group compared to the regular plate group. There was no significant difference in Safranin $\mathrm{O}$ staining in the sliding plate group and the control group at either 4 or 8 weeks after surgery. In the regular plate group, the Safranin O staining exhibited a lighter color in animals at either 4 and 8 weeks after surgery (Fig. 5).

Table I. Histological data of experimental goats

\begin{tabular}{llll}
\hline EPT ( $\mu \mathrm{m})$ & $\begin{array}{l}\text { Postoperative } 4 \\
\text { weeks (range) }\end{array}$ & $\begin{array}{l}\text { Postoperative 8 } \\
\text { weeks (range) }\end{array}$ & $\begin{array}{l}\text { P } \\
\text { value }\end{array}$ \\
SPG & $\begin{array}{l}962.19 \pm 20.89 \\
(937.96-989.75)\end{array}$ & $\begin{array}{l}968.09 \pm 16.63 \\
(940.61-984.87)\end{array}$ & $>0.05$ \\
RPG & $\begin{array}{l}824.87 \pm 21.2 \\
(793.70-850.07)^{*}\end{array}$ & $\begin{array}{l}774.95 \pm 18.2 \\
(753.85-792.19)^{*}\end{array}$ & $<0.05$ \\
CG & $981.02 \pm 9.86$ & $986.74 \pm 10.01$ & $>0.05$ \\
PCC(cells/mm) & $(970.71-996.18)$ & $(972.16-997.93)$ & \\
SPG & $7.5 \pm 1.05(6-9)$ & $7.33 \pm 0.82(6-8)$ & $>0.05$ \\
RPG & $5.67 \pm 0.82(5-7)^{*}$ & $4.67 \pm 0.82(4-6)^{*}$ & $<0.05$ \\
CG & $8.17 \pm 0.75(7-9)$ & $7.83 \pm 0.75(7-9)$ & $>0.05$ \\
HCC(cells/mm) & & & \\
SPG & $6.33 \pm 0.82(5-7)$ & $5.83 \pm 0.75(5-7)$ & $>0.05$ \\
RPG & $4.67 \pm 0.82(4-6)^{*}$ & $3.67 \pm 0.82(3-5)^{*}$ & $<0.05$ \\
CG & $7.17 \pm 0.75(6-8)$ & $6.67 \pm 0.82(6-8)$ & $>0.05$ \\
\hline
\end{tabular}

Comparison between SPG and RPG, * $p<0.05$; CG: control group; EPT: epiphyseal plate thicknesses; HCC: hypertrophic cell counts; PCC: proliferation cell counts; RPG: regular plate group; SPG: sliding plate group. 

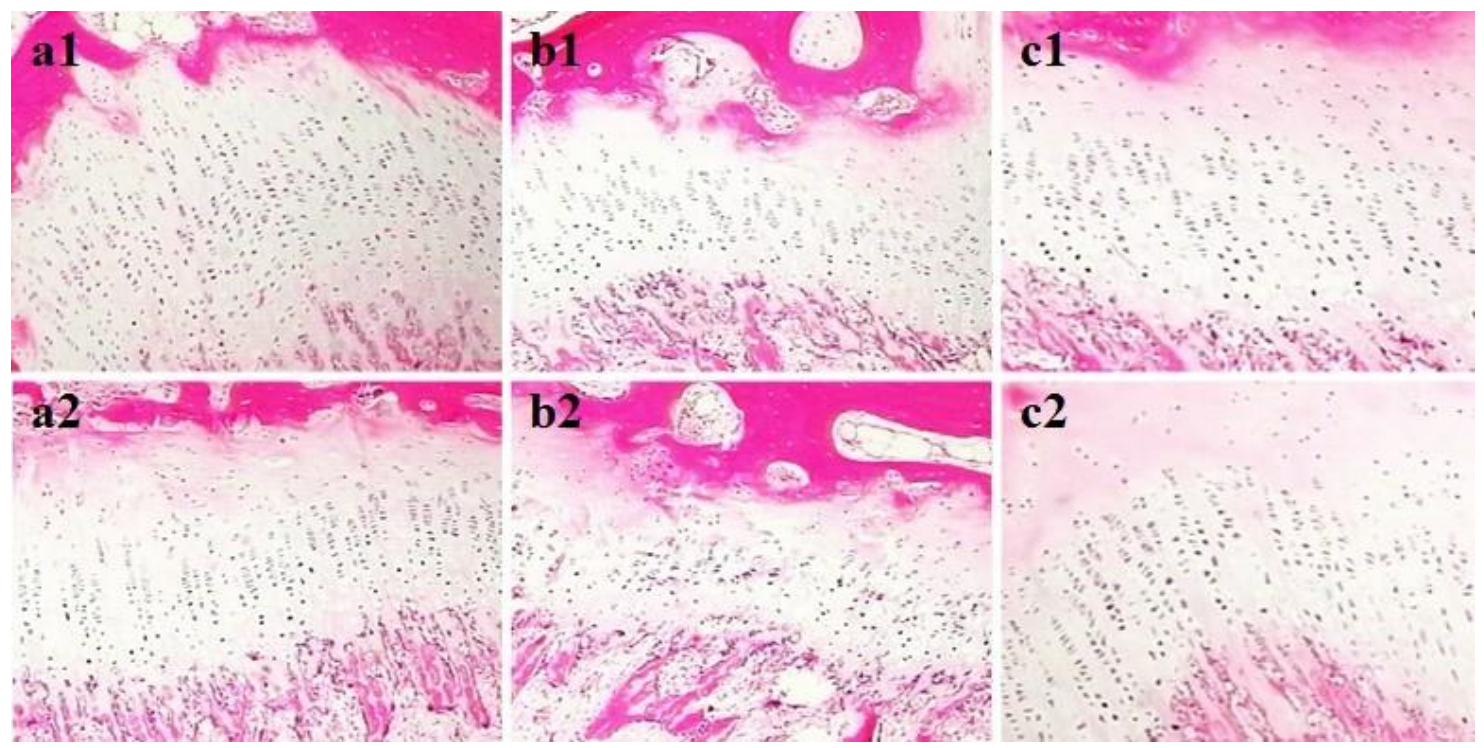

c2

Fig. 4 Compared to the regular plate group, the sliding plate group exhibited more normal epiphyseal growth and histologic features, and there were no significant difference between the sliding plate group and the control group. al: HE staining of epiphyseal plate from the sliding plate group at 4 weeks after surgery (40x). a2: HE staining of epiphyseal plate from the sliding plate group at 8 weeks after surgery (40x). bl: HE staining of epiphyseal plate from regular plate group at 4 weeks after surgery (40x). b2: HE staining of epiphyseal plate from regular plate group at 8 weeks after surgery (40x). cl: $\mathrm{HE}$ staining of epiphyseal plate from control group at 4 weeks after surgery (40x). c2: HE staining of epiphyseal plate from control group at 8 weeks after surgery (40x).
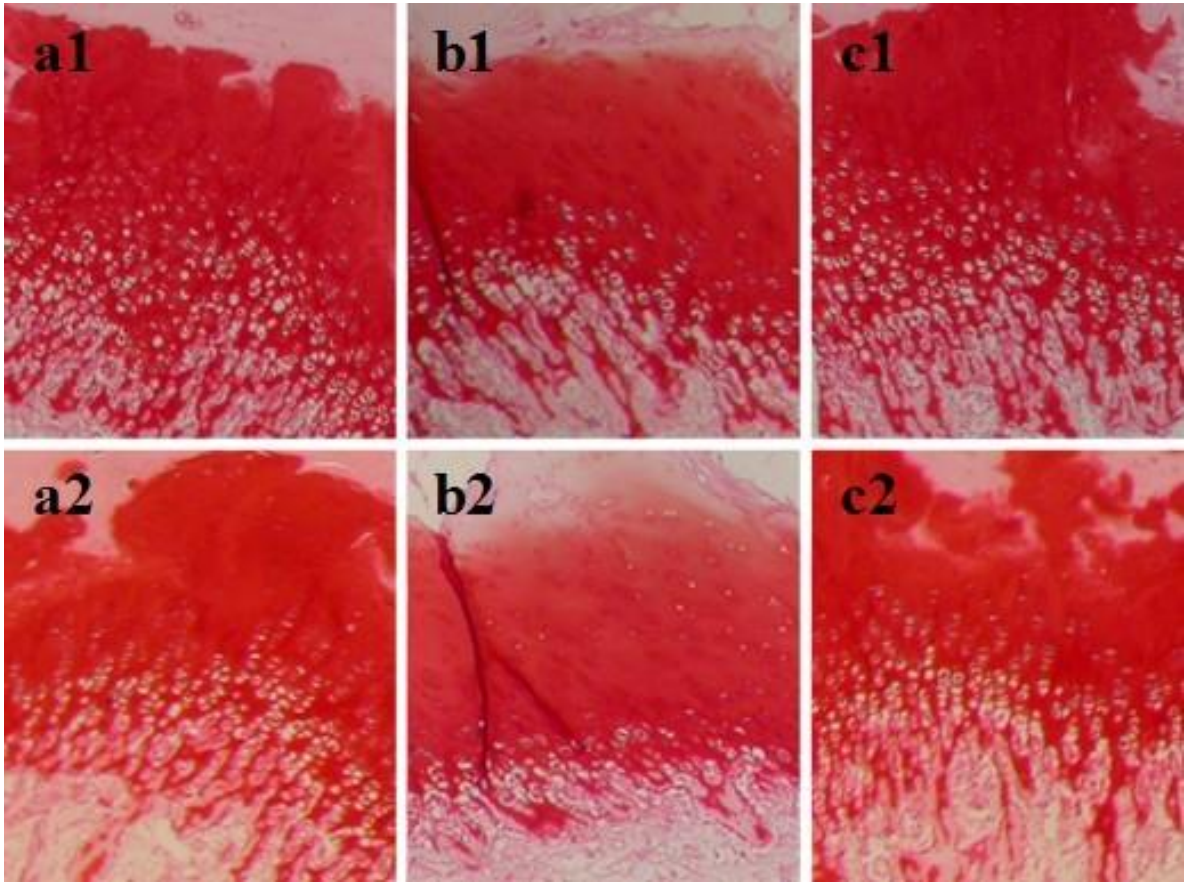

Fig. 5 Safranin O staining was significantly stronger in the epiphyseal plates from the sliding plate group compared to the regular plate group, and there were no significant difference between the sliding plate group and the control group. al: Safranin $O$ staining of epiphyseal plate from the sliding plate group at 4 weeks after surgery (40x). a2: Safranin $O$ staining of epiphyseal plate from the sliding plate group at 8 weeks after surgery $(40 \mathrm{x})$. bl: Safranin $\mathrm{O}$ staining of epiphyseal plate from regular plate group at 4 weeks after surgery (40x). b2: Safranin $O$ staining of epiphyseal plate from regular plate group at 8 weeks after surgery (40x). cl: Safranin O staining of epiphyseal plate from control group at 4 weeks after surgery (40x). c2: Safranin $O$ staining of epiphyseal plate from control group at 8 weeks after surgery (40x). 


\section{Discussion}

Internal fixation methods employed to treat distal femoral epiphyseal fractures in children include external fixation [10], screws [11], Kirschner wires [11], flexible intramedullary nailing [12], and regular plate fixation $[13,14]$, etc. Each method has its advantages and disadvantages, and some degree of disturbance in longitudinal femoral growth may occur, according to the severity of the original injury or surgical trauma and method chosen. A better prognosis is obtained with fixation techniques that do not compress the growth plate.

For complex fractures of the distal femoral growth plate, it is difficult to fix and recover the fracture by using screws, Kirschner wires or flexible intramedullary nailing. Regular plate fixation can provide reliable fixation, but it can not be taken out in time. As the epiphyseal plate grows, the steel plate produces a vertical force which is reverse in direction to epiphyseal growth. This mechanical compression force restricts epiphyseal growth and retards normal bone growth [15-17]. In order to overcome the drawbacks, we designed the sliding plate which is conjectured to slide with epiphyseal growth, while simultaneously providing reliable fixation of bone fractures. Therefore the sliding plate avoids restriction of epiphyseal growth, and greatly reduces the risk of complications like epiphyseal growth retardation, epiphyseal premature closure and angular deformity.

The sliding plate is a transformation based on traditional anatomical plate. To realize the vertical slide function, the sliding plate is made of two parts. The head part is used to fix the femoral condyle, and the body part is used to fix the diaphysis. The head part has a drawer-like slot along which the body part can slide. The biomechanical design of the sliding plate enables it enough strength in anti-compression, anti-bending, and anti-torsion. The strength and stability of the sliding plate comes from its firm drawer-like combination which is strengthened at the overlap of two parts, and from its stable screw-plate locking design. Examination of imaging studies performed on the present study indicated that in contrast to the regular plates, the sliding plates can extend in concert with epiphyseal growth. In addition, using light microscope examination and histochemical analysis with Safranin O, we confirmed that the sliding plates do not suppress epiphyseal growth, thus potentially reducing secondary epiphyseal injury and preventing epiphyseal growth retardation and premature closure.

When applying the sliding plate for distal femoral epiphyseal fractures, we should pay attention in following aspects: the head part and body part of the sliding plate should be united to the utmost extent to avoid their compressing slide in vertical direction, which can exert compressive stress on the fracture and impair epiphyseal growth, and the screws of the body part should be embedded completely into the iron plate so that it can not get the head part stuck and failing to slide.

\section{Conclusion}

It was possible to conclude that the sliding plate allows longitudinal bone growth, without blocking the distal femur growth plate if it is appropriately placed. Although these findings cannot be directly extrapolated to treatment of distal femoral epiphyseal fractures in children, they form a useful basis for future studies.

\section{Acknowledgments}

This work was supported by the key projects from Nanjing Military Region during the $11^{\text {th }}$ Five-Year Plan Period (No.06Z30).

\section{Conflict of Interest}

The authors have declared that no conflict of interest exists.

\section{References}

1. Gomes LS, Volpon JB, Goncalves RP. Traumatic separation of epiphyses. An experimental study in rats. Clin Orthop Relat Res. 1988; 236:286-295.

2. Greco F, de Palma L, Specchia N, Mannarini M. Growth-plate cartilage metabolic response to mechanical stress. J Pediatr Orthop. 1989; 9:520-524.

3. LeVeau BF, Bernhardt DB. Developmental biomechanics. Effect of forces on the growth, development, and maintenance of the human body. Phys Ther. 1984; 64:1874-1882.

4. Graham JM, Gross RH. Distal femoral physeal problem fractures. Clin Orthop Relat Res. 1990; 255:51-53.

5. Peterson HA, Madhok R, Benson JT, IIstrup DM, Melton LJ 3rd. Physeal fractures: part 1. Epidemiology in Olmsted County, Minnesota, 1979-1988. J Pediatr Orthop. 1994; 14:423-430.

6. Salter RB, Harris WR. Injuries involving the epiphyseal plate. J Bone Joint Surg Am. 1963; 45: 587-622.

7. Inoue T, Naito M, Fujii T, Akiyoshi Y, Yoshimura I, Takamura K. Partial physeal growth arrest treated by bridge resection and artificial dura substitute interposition. J Pediatr Orthop B. 2006; 15:65-69.

8. Radhakrishnan P, Lewis NT, Mao JJ. Zone-specific micromechanical properties of the extracellular matrices of growth plate cartilage. Ann Biomed Eng. 2004; 32:284-291.

9. Hedequist D, Bishop J, Hresko T. Locking plate fixation for pediatric femur fractures. J Pediatr Orthop. 2008; 28:6-9.

10. Hedin H, Hjorth K, Rehnberg L, Larsson S. External fixation of displaced femoral shaft fractures in children: a consecutive study of 98 fractures. J Orthop Trauma. 2003; 17:250-6.

11. Flynn JM, Skaggs DL, Sponseller PD, Ganley TJ, Kay RM, Leitch KK. The surgical management of pediatric fractures of the lower extremity. Instr Course Lect. 2003; 52:647-59. 
12. Metaizeau JP. Stable elastic intramedullary nailing for fractures of the femur in children. J Bone Joint Surg Br. 2004; 86:954-7.

13. Caird MS, Mueller KA, Puryear A, Farley FA. Compression plating of pediatric femoral shaft fractures. J Pediatr Orthop. 2003; 23:448-52.

14. Sink EL, Hedequist D, Morgan SJ, Hresko T. Results and technique of unstable pediatric femoral fractures treated with submuscular bridge plating. J Pediatr Orthop. 2006; 26:177-81.

15. Villemure I, Stokes IA. Growth plate mechanics and mechanobiology. A survey of present understanding. J Biomech. 2009; 42:1793-1803.

16. Stokes IA, Mente PL, Iatridis JC, Farnum CE, Aronsson DD. Enlargement of growth plate chondrocytes modulated by sustained mechanical loading. J Bone Joint Surg Am. 2002; 84A:1842-1848.

17. Stokes IA, Clark KC, Farnum CE, Aronsson DD. Alterations in the growth plate associated with growth modulation by sustained compression or distraction. Bone. 2007; 41:197-205. 